\title{
Influenza infections during the period of 2019-2020 -just before the COVID-19 outbreak- in a single unit
}

Şule Gökçe ${ }^{1 *}$, Feyza Koç², Candan Çiçek ${ }^{3}$, Humay Mehdiyeva ${ }^{1}$, Aslı Aslan ${ }^{2}$

${ }^{1}$ Ege University Faculty of Medicine, Department of Pediatrics, General Pediatrics Unit. Ege University, Bornova, 35040, Izmir, Turkey.

${ }^{2}$ Associated Professor Doctor, Ege University Faculty of Medicine, Department of Pediatrics, General Pediatrics Unit. Ege University, Bornova, 35040, Izmir, Turkey.

${ }^{3}$ Professor Doctor, Ege University Faculty of Medicine, Department of Microbiology. Ege University, Bornova, 35040, Izmir, Turkey.

*Corresponding Author: Şule Gökçe, Ege University Faculty of Medicine, Department of Pediatrics, General Pediatrics Unit. Ege University, Bornova, 35040, Izmir, Turkey.

Received date: February 25, 2021; Accepted date: April 02, 2021; Published date: April 06,2021

Citation: Ş Gökçe, F Koç, C Çiçek, H Mehdiyeva, A Aslan. (2021) Influenza infections during the period of 2019-2020 -just before the COVID19 outbreak- in a single unit. Journal of Clinical and Laboratory Research. 2(2) DOI: 10.31579/ 2768-0487 /011

Copyright: (02021 Şule Gökçe. This is an open-access article distributed under the terms of the Creative Commons Attribution License, which permits unrestricted use, distribution, and reproduction in any medium, provided the original author and source are credited.

\section{Abstract}

Background and Purpose: We aimed to evaluate the demographic features, clinical manifestations, course of disease, and outcome of children with Influenza and Influenza complications.

Methods: In the winter season -just before the COVID 19 outbreak- between October 2019 and March 2020, the cases with Influenza diagnosed by rt-PCR (real time polymerase chain reaction) requiring hospitalization retrospectively were evaluated with hospital records.

Results: A total 36 children were hospitalized with Influenza virus infection. The most frequent presentations of the subjects were malaise and fever. Other clinical presentations consisted of vomitting (36.1\%), abdominal pain $(30.5 \%)$, myalgia $(27.7 \%)$, diarrhea $(25 \%)$, dry cough $(19.4 \%)$, and arthralgia $(11.1 \%)$. There were respiratory distress in 19 patients $(52.7 \%)$ in our cohort. The most frequent underlying disease requiring hospitalization was neurological diseases.

Conclusions and Recommendation: The Influenza infection was associated with a wide variety of clinical findings during the hospital admission in pediatric patients. Immunization programs for Influenza should be considered for all children aged $>6$ months, and should be also targeted the pediatric population that likely to be hospitalized with predisposing medical conditions- particularly neurological- every influenza season. Encouraging the use of Influenza vaccines will provide a decrease in Influenza that have similar presentations of COVID-19.

Keywords: influenza; influenza infections; children; clinical characteristics; immunization

\section{Introduction}

Influenza (flu) is an acute respiratory tract disease caused by Influenza viruses $\mathrm{A}$ and $\mathrm{B}$ ( $\operatorname{Inf} \mathrm{A}$ and $\mathrm{B}$ ), and is generally responsible for outbreaks and epidemics worldwide in the winter seasons. The disease is a contagious disease that usually characterized by an acute onset upper airway tract symptoms including fever, cough, rhinitis, headache, myalgia in a healthy person. However, there have been many reports mentioning the association between flu and morbidity/mortality, especially in children, the elderly, pregnant women, health workers, and those with serious medical conditions. According to estimates of the World Health Organization (WHO), the virus effects the people as $16-28 \%$ in preschool-aged children, as 34-43\% in school-aged children, and 5-10\% of adults every winter season $[1,2]$. Despite the fact that the Influenza infections generally cause an uncomplicated disease, some pediatric patients might become severely ill, to the point of hospitalization or even death; particularly having a neurological condition, immunosuppression, an abnormal chest radiograph, and moderate persistent asthma [3, 4]. In order to prevent complications and deaths, the Influenza vaccine in pediatric age from 6 months to 5 years has been recommended by the WHO.

There are four types of Influenza viruses including A, B, C and D; seasonal influenza viruses ( Inf $\mathrm{A}$ and $\mathrm{B}$ ) that spread from person to person, through contact with respiratory secretions or contaminated objects are responsible for outbreaks each year. Inf A viruses are further classified into subtypes according to the proteins known as the combinations of hemagglutinin (HA) and the neuraminidase (NA) on the surface of the virus. The subtype $\mathrm{A} / \mathrm{H} 1 \mathrm{~N} 1$ and $\mathrm{A} / \mathrm{H} 3 \mathrm{~N} 2$ Influenza viruses had been circulating since the 1900s, and the last efficacious (written as $\mathrm{A}(\mathrm{H} 1 \mathrm{~N} 1)$ pdm09) outbreak of the respiratory tract disease was first noticed in Mexico. It has been declared by the WHO as a pandemic illness originated from A/H1N1 in June 2009 [1]. 
The classic symptoms of simple influenza virus infection include abrupt onset of fever, headache, myalgia, accompanied by features of respiratory-tract illness, such as cough, sore throat, and rhinitis $[5,6]$. The presence of multiplex polymerase chain reaction (PCR) has provided a permitting simultaneous amplification of the viruses and allowed to examine the epidemiological differences/clinical characteristics of the viruses [7]. Due to the fact that the economic burden of Influenza effects the health services and the insurance and legal systems; many studies have been focusing for many years on Influenza virus and its outbreaks to determine the behavior; for prevention and treatment strategies against it.

This study aimed to analyze the epidemiological and clinical features as well as laboratory findings and outcome of children admitted to a tertiary pediatric hospital during the period of 2019-2020 winter season with confirmed Influenza virus infection. The index study also purposed to detect whether there were any differences between Inf A and B in the presentation, hospital course, and outcome of pediatric patients.

\section{Method and Materials}

Study Design and Period: This retrospective, cross-sectional, observational study has evaluated the patients (aged from 2 months-18 years) diagnosed with Influenza or been thought a preliminary diagnosis of Influenza at the Ege University Medical Faculty General Pediatrics ward of Children's Hospital between October 2019 and March 2020. This study was approved by the ethics committee of Ege University and the institutional review board of Ege University Children's Hospital (209T/39).

The Detection of Influenza viruses: A nasal smear was obtained from each patient and transported to the laboratory in a viral universal transport medium (Copan Diagnostics, Brescia, Italy). The extraction of nucleic acids was performed on a fully automated device with EZ1 Virus Mini Kit v2 (Qiagen, Luxembourg) according to the manufacturer's recommendations. Influenza viruses were identified by real-time polymerase chain reaction method (RealAccurate, Respiratory RT PCR, PathoFinder, Netherlands, and Seeplex RV15 ACE Detection, Seegene, South Korea) using FTD Respiratory pathogens 21 ov kit (Fast Track
Diagnosis, Luxembourg) and the results were obtained from the RotorGene (Qiagen, Luxembourg) device.

Patients: A structured case report form was filled by the physician from the parents of children with influenza infection for clinical, epidemiological, and laboratory data. The case report forms have covered the data such as gender, age, pre-existing chronic disease, date of symptom onset and date of hospital admission, complications (pneumonia, acute respiratory distress syndrome, multiorgan failure, and septic shock), antiviral treatment received, seasonal Influenza vaccination status, and laboratory data included confirmed virus type, and several biochemical analyze results.

Statistical analyses: Data analyses were performed using SPSS version 21.0. The quantitative data including percentage, mean, and standard deviations were calculated by simple descriptive analyses. Shapiro-Wilk test was used to check normality and according to the results parametric or nonparametric suitable statistical tests were performed. Differences in the distribution of categorical parameters were analyzed by $\chi 2$ or Fisher's Exact Test. An independent samples T-test or nonparametric alternative of its Mann-Whitney $U$ test was used to compare 2 groups for a quantitative variable. The differences between Influenza subtypes were assessed and a P-value of $<0.05$ was considered to be significant.

\section{Results}

Patients: During the winter season between October 2019-March 2020, a total of 440 patients have been hospitalized for any disease at General Pediatrics Unit. Thirty-six $(8.1 \%)$ cases with Influenza requiring hospitalization were evaluated in the study. Of the 36 hospitalized cases, $77.7 \%$ (28 patients) tested positive for Inf A virus and 22.3\% (8 patients) for Inf B. The most frequent A subtypes were H1N1 (66.7\%); H3N2 was accounted as 4 patients (11.1\%). Table 1 has shown the demographic, clinical and presenting features of children with Influenza. Of the total, $55.6 \%$ were male, $44.4 \%$ were female. The median length of hospital stay was 4 days (3) in children with Influenza viruses. The most common presentations on admission were: malaise in $31(86.1 \%)$, fever in 30 $(83.3 \%)$, and sore throat in $15(41.6 \%)$ cases.

\begin{tabular}{|l|r|}
\hline Demografic characteristics & $25(63.7)$ \\
\hline Age, months, [median, (IQR)] & $7(19.4)$ \\
\hline Age distributions, n (\%) & $5(13.9)$ \\
\hline $2-6$ & $14(38.9)$ \\
\hline $7-12$ & $10(27.8)$ \\
\hline $12-60$ & $20(55.6)$ \\
\hline$>60$ & $16(44.4)$ \\
\hline Gender, n (\%) & $21(58.3)$ \\
\hline Male & $10(27.8)$ \\
\hline Memale & $4(11.1)$ \\
\hline January & $1(2.8)$ \\
\hline December & \\
\hline February & $31(86.1)$ \\
\hline November & $30(83.3)$ \\
\hline Clinical features and presenting symptoms, $\mathrm{n}(\%)$ & $15(41.6)$ \\
\hline Malaise & $13(36.1)$ \\
\hline Fever & $13(36.1)$ \\
\hline Sore throat & $11(30.5)$ \\
\hline Rhinorrhea & \\
\hline Vomitting & \\
\hline Abdominal pain & \\
\hline & \\
\hline
\end{tabular}




\begin{tabular}{|l|r|}
\hline Myalgia & $10(27.7)$ \\
\hline Diarrhea & $9(25)$ \\
\hline Dry cough & $7(19.4)$ \\
\hline Arthralgia & $4(11.1)$ \\
\hline Respiratory distress & $2(5.6)$ \\
\hline Mild & $11(30.6)$ \\
\hline Moderete & $6(16.7)$ \\
\hline Severe & $4(3)$ \\
\hline LOS, days [median (IQR)] & $0(0)$ \\
\hline Influenza vaccination status, n (\%) & \\
\hline
\end{tabular}

IQR; interquartile range, LOS; Length of stay in hospital

Table 1: Demographic characteristics and clinical presentations of the patients with Influenza

Laboratory and Clinical follow-up: Table 2 has shown the treatment modalities, hematological findings, complications, and other features of the subjects. Oseltamivir was administered in 16 (44.4\%) children. A total of $9(25 \%)$ children were given antibiotics together with antiviral drugs. $44.4 \%$ received antiviral treatment with oseltamivir, of whom $56.3 \%$ received it within 48 hours after symptom onset. The median time between symptom onset and hospital admission was 3 (2) days. The most frequent complication was lower respiratory tract infection (LRTI) 19 (52.7\%), of whom one patient diagnosed with acute respiratory distress syndrome $(5.2 \%)$ and of whom 3 patients needed mechanical ventilation. The median white blood cell count was 7.5
$(8.9) / \mathrm{mm}^{3}$; the median absolute neutrophil count was $3.4(5.9) / \mathrm{mm}^{3}$; neutrophile count as $<1500 / \mathrm{mm}^{3}$ was seen in $10(27.8 \%)$ patients. Creatine kinase was measured in 13/36 children, with the mean value of 1894 (max: 12.367; min: 19) U/L; of whom six cases were diagnosed with myositis. Two patients with Inf A; 1 patient with Inf B (8.3\%) cases required pediatric intensive care unit. In terms of the chronic conditions and predisposing factors; at least one underlying medical condition was detected for 13 (43\%) of the 36 children; neurological disorders were the most frequent condition in our study cohort $(n=5,38.4 \%$ of all 13 children with predisposing factors).

\begin{tabular}{|c|c|}
\hline \multicolumn{2}{|l|}{ Treatment Modalities and Complications, n (\%) } \\
\hline Only antibiotherapy & $17(47.2)$ \\
\hline Only antiviral treatment (oseltamivir) & $16(44.4)$ \\
\hline$\leq 48 \mathrm{~h}$ & $9(56.3)$ \\
\hline After $48 \mathrm{~h}$ & $7(43.7)$ \\
\hline Both antibiotherapy and antiviral treatment & $9(25)$ \\
\hline $\mathrm{O}_{2}$ requirement & $18(50)$ \\
\hline Only Oxygen treatment & $13(73.6)$ \\
\hline Other & $2(10.5)$ \\
\hline Respiratory support (Mechanical ventilation) & $3(15.7)$ \\
\hline \multicolumn{2}{|l|}{ Complications, n (\%) } \\
\hline LRTI & $19(52.7)$ \\
\hline Pneumonia & $14(73.6)$ \\
\hline Bronchiolitis & $5(37.4)$ \\
\hline Hepatitis & $9(25)$ \\
\hline Myositis & $6(16.6)$ \\
\hline Bacterial coinfection (Pneumonia) & $5(13.8)$ \\
\hline Sepsis & $2(5.5)$ \\
\hline Renal dysfunction & $2(5.5)$ \\
\hline Vasculitis & $1(2.7)$ \\
\hline Acute respiratory distress syndrome & $1(2.7)$ \\
\hline \multicolumn{2}{|c|}{ Co-morbidity or diagnosed during hospitalization $(\mathrm{n}=13), \mathrm{n}(\%)$} \\
\hline Neurological disease & $5(38.4)$ \\
\hline Obesity & $2(15.2)$ \\
\hline Hydronephrosis & $2(15.2)$ \\
\hline Down syndrome+septal defect & $1(7.8)$ \\
\hline Hematological disease & $1(7.8)$ \\
\hline Immunological disease & $1(7.8)$ \\
\hline D vitamin deficiency & $1(7.8)$ \\
\hline \multicolumn{2}{|l|}{ Laboratory and radiological findings } \\
\hline White blood cell, [median (IQR)] cells $/ \mathrm{mm}^{3}$ & $7.5(8.9)$ \\
\hline Lymphocyte, [median (IQR)], (\%) & $62(69)$ \\
\hline Neutrophil count, [median (IQR)] cells $/ \mathrm{mm}^{3}$ & $3.4(5.9)$ \\
\hline \multicolumn{2}{|l|}{ Neutrophil status, n (\%) } \\
\hline Normal & $26(72.2)$ \\
\hline
\end{tabular}




\begin{tabular}{|c|c|}
\hline Neutropenia & $10(27.8)$ \\
\hline C-reactive protein, [median (IQR)] mg/l & $12.8(2.6)$ \\
\hline \multicolumn{2}{|l|}{ X-ray findings, $(n=13), n(\%)$} \\
\hline Interstitial pneumonia & $11(84.6)$ \\
\hline Lobuler involvement & $2(15.4)$ \\
\hline \multicolumn{2}{|l|}{ Co-infection, $(n=4)$, n (\%) } \\
\hline Coronavirus (Not COVID-19) & $2(50)$ \\
\hline Norovirus & $1(25)$ \\
\hline RSV A+RSV B & $1(25)$ \\
\hline
\end{tabular}

IQR; interquartile range, LRTI; Lower respiratory tract infection, RSV; Respiratory syncytial virus, COVID-19; Coronavirus disease 2019

\section{Table 2: Treatment modalities, complications, and laboratory/radiological measurement of the patients hospitalized with Influenza}

Clinic and demographical differences (Table 3): The cases of Inf A were younger $(\mathrm{P}>0.05)$. No differences were found according to sex $(\mathrm{P}>0.05)$. There was no difference in the frequency of the gastrointestinal symptoms of nausea and/or vomiting with either Inf A or B. Among the Inf A group, 2 patients presented with a febrile seizure.
With regard to other presentations; 5 cases with myositis have admitted to our clinic in Inf A group, 2 in the Inf B group. There were also no significant differences in the symptoms and other features according to virus type.

\begin{tabular}{|c|c|c|c|}
\hline & $\begin{array}{r}\text { Influenza } A^{¥} \\
(n=28)\end{array}$ & $\begin{array}{r}\text { Influenza B } \\
(n=8)\end{array}$ & $P$ value \\
\hline Age, months, [median, (IQR)] & $19(39.5)$ & $83(97.5)$ & $0.083^{*}$ \\
\hline Age distributions, n (\%) & & & $0.087 * * *$ \\
\hline $2-6$ months, & $6(21.4)$ & $1(12.5)$ & \\
\hline 7-12 months, & $4(14.3)$ & $1(12.5)$ & \\
\hline 12-60 months, & $13(46.4)$ & $1(12.5)$ & \\
\hline$>60$ months, & $5(17.9)$ & $5(62.5)$ & \\
\hline $\begin{array}{l}\text { Gender, n (\%) } \\
\text { Male } \\
\text { Female }\end{array}$ & $\begin{array}{l}13(46.4) \\
15(53.6)\end{array}$ & $\begin{array}{l}7(87.5) \\
1(12.5)\end{array}$ & $0.053 * *$ \\
\hline \multicolumn{4}{|l|}{ Symptoms, n (\%) } \\
\hline Fever & $25(89.3)$ & $5(62.5)$ & $0.073 * * *$ \\
\hline Malaise & $25(89.3)$ & $6(75)$ & $0.303 * * *$ \\
\hline Myalgia & $6(21.4)$ & $4(50)$ & $0.112 * * *$ \\
\hline Cough & $4(14.3)$ & $3(37.5)$ & $0.167 * *$ \\
\hline Respiratory distress & & & $0.772 * *$ \\
\hline Mild & $2(7.1)$ & $0(0)$ & \\
\hline Moderate & $9(32.1)$ & $2(25)$ & \\
\hline Severe & $4(14.3)$ & $2(25)$ & \\
\hline Diarrhea & $8(28.6)$ & $1(12.5)$ & $0.648 * *$ \\
\hline Vomitting & $11(39.3)$ & $2(25)$ & $0.883 * *$ \\
\hline \multicolumn{4}{|l|}{ Laboratory and radiological findings } \\
\hline White blood cell, [median (IQR)] $/ \mathrm{mm}^{3}$ & $7.5(8.1)$ & $8.2(10.6)$ & $0.703 *$ \\
\hline Lymphocyte, [median (IQR)], (\%) & $64(62)$ & $59(61)$ & $0.827 *$ \\
\hline Neutrophil count, [median (IQR)] $/ \mathrm{mm}^{3}$ & $4(5.4)$ & $2.1(9.9)$ & $0.581^{*}$ \\
\hline Neutrophil status, n (\%) & & & $0.112 * *$ \\
\hline Normal & $22(78.6)$ & $4(50)$ & \\
\hline Neutropenia & $6(21.4)$ & $4(50)$ & \\
\hline LOS, days [median (IQR)] & $4(4)$ & $4(1.5)$ & $0.969 *$ \\
\hline
\end{tabular}

LOS; Length of stay in hospital, IQR; Interquartile range *Mann-Whitney U, **Fisher's Exact Test, *** Chi-square Test ${ }^{\ddagger} \mathrm{A} / \mathrm{H} 1 \mathrm{~N} 1=24$ patients; $\mathrm{A} / \mathrm{H} 3 \mathrm{~N} 2=4$ patients

\section{Table 3: Comparison of the characteristics of subjects according to the type of Influenza virus}

\section{Discussion}

The study has shown the clinical manifestations of Influenza infections among hospitalized children with PCR-confirmed Influenza in the period of October 2019-March 2020 in the General Pediatrics ward of Children's Hospital. The most prevalent type of Influenza in children was Inf A (77.7\%); it was more common than Inf B/ non-H1N1 (22.3\%). With regard to the age distribution, Inf A was younger than those with Inf B. It has been reported that age could be a significant differential factor by Livia Mancinelli et al [8]; when they analyzed the virus Inf A was more common in children less than 12 months, and Inf B was more prevalent in school-age. Similarly, a study conducted from Canada stated that a patient with Influenza under 12 months of age that results in a 2.3 times required the hospitalization [9]. Another study has reported that the Inf A (H1N1) pdm09 subtype was more common and was associated with severe clinical features in children aged $<2$ years; however, Inf B infection was more frequently seen in older ages [10]. It was found that hospitalized children were generally preschool-aged, with a median age of around 25 months. Influenza A subtype was more common in subjects aged under 24 months (63.1\%), of whom needed intensive care unit and mechanical 
ventilation. The children $>5$ years (median 83 months) infected with Influenza B were more frequent in this study. In contrast, a study conducted from the USA showed that the median age of pediatric patients hospitalized with Influenza A were 6.5 years, whereas; this age in Inf B was 1.3 years [11].

As reported previously, Influenza virus infection is most common in previously healthy children with fever and nonspecific upper airway tract signs and symptoms that are similar to those of other infectious processes in hospitalized children. In the study population, the clinical course was mostly self-limited with malaise $(86.1 \%)$, fever $(83.3 \%)$, and sore throat $(41.6 \%)$ as predominant symptoms. In literature, malaise and fever are the most prominent manifestation and gastrointestinal symptoms were found to be more prevelant in Inf $\mathrm{A} / \mathrm{H} 1 \mathrm{~N} 1$ than other influenza types [11]. Although there was no significant association to a certain Influenza strain; compitable with the literature, our subjects with Inf A presented with gastrointestinal symptoms like vomiting and diarrhea were more common than those Inf B. No consistent differences were observed in the other clinical presentation of patients by viral subtype in the study. The risk of complications has been reported in $25 \%$ to $49 \%$ in the children with underlying diseases [12]. Neurological disorders were the most common disease referring principal risk factor accounting for $38.4 \%$ of all Influenza positive cases with several diseases.

It was found that Influenza infected patients had higher rates of LRTI (19/36 cases), as observed in other studies. Mireia Jane et al [10] have evaluated a total of 291 patients; $62.2 \%$ of cases had pneumonia, $26.8 \%$ acute respiratory distress syndrome, and $11.7 \%$ bacterial pneumonia during the Inf A and B infection. A retrospective observational study was conducted in 360 cases of whom $39.7 \%$ of patients suffered from LRTI; pneumonia and acute respiratory failure [13]. The children hospitalized with laboratory-confirmed $\mathrm{A} / \mathrm{H} 1 \mathrm{~N} 1$ were reviewed by $\mathrm{A}$. Desiree LaBeaud [14]; of the 47 severe cases, 34 required oxygen, 20 required intubation, 7 had acute respiratory distress syndrome and 23 cases with pneumonia. Many patients with Influenza A/H1N1 manifested with gastroenteritis without respiratory symptoms and they required hospitalization due to dehydration and inability to feed in our study. So, it might be easily declared that the clinicians should also consider the Influenza infections presenting gastrointestinal symptoms in the setting of the Influenza season. In the laboratory, it has been found statistically insignificant lower absolute neutrophil counts, particularly in children infected with Inf B compared with Inf A/non-H1N1 in this study. A research stated by Ching-Fen Shen et al [15] have reported that the lower leucocyte counts in pediatric patients with Inf B compared with Inf $\mathrm{A} /$ non-H1N1 and decreased leukocytes might be used criteria for the diagnosis of Inf B infection before viral culture results.

There has been recommended by the World Health Organization and Centers for Disease Control and Prevention (CDC) that children should be vaccinated between 6 months and 5 years to help avoid hospital admissions for Influenza. There is also a recommendation for children between 6 months and three years of age the implementing the booster dose [16]. On February 11, 2020, the World Health Organization officially described a pandemic novel virus named Coronavirus disease 2019 (COVID-19), and the International Committee on Taxonomy of Viruses has called it severe acute respiratory syndrome Coronavirus 2 (SARS-CoV-2). The clinical manifestations of Novel coronavirus infection consist of fever, cough, myalgia, and/or fatigue. Due to the fact that the possibility of Influenza viruses and COVID-19 virus share the same season and have quite similar manifestations, there is an anxiousness whether to be accurately identified these two respiratory viral infections. Although it has been reported that there were many differences in clinical presentations such as lower severity illness scores at presentation and mortality in sufferer with $\mathrm{A} / \mathrm{H} 1 \mathrm{~N} 1$ compared with those infected with COVID-19, it is thought to be difficult to differentiate these two diseases upcoming winter [17].

Potential limitations can be listed: the study may not reflect incidence of actual Influenza due to only patients with laboratory-confirmed Influenza, unable to collect information about the clinical cases in patients admitted to the outpatient clinic and emergency department and finally, fairly small study size may also have restricted the identification of significant relations with the disease predictors and severity.

In this study- just before the declarations of COVID-19 pandemic - we have described that the clinical features of children with Influenza during the 2019-2020 season and defined whether there was differences. Pediatric patients were presented with quite a variety of clinical findings. The neurological disorder has over again been confirmed as the most significant and prevelant risk factor for Influenza infection in children. It should not to be neglected the vaccination against Influenza for all children, especially children with a history of chronic conditions. There could be a vigorous possibility that the children could have a co-infection (Influenza and COVID-19) and we have never known this co-existance. Encouraging the use of Influenza vaccines will provide a decrease in Influenza infection that have similar presentations of COVID-19.

\section{Conflıct of Interests}

The author(s) declared no potential conflicts of interests with respect to the authorship and/or publication of this article.

\section{Authors' Contributions}

ŞG: conceived of the study, and participated in its design and coordination and drafted the manuscript. ŞG and FK: participated in the design of the study, and collection and acquisition of data. FK: helped to the design of the study, collection and acquisition of data, and helped to draft the manuscript. ŞG: general supervision of the research group, coordination and helped to draft the manuscript. CÇ: carried out molecular analysis. ŞG: performed the statistical analysis. All authors read and approved the final manuscrip

\section{Funding}

The author(s) received no financial support for the research and/or authorship of this article.

\section{Ethics}

The study was approved by the institutional review board named The Ethics Committee of Ege University (20-9T/39).

\section{References}

1. World Health Organization. (2011) World now at the start of 2009 influenza pandemic.

2. Kelly H, Peck HA, Laurie KL, et al. (2011) The age-specific cumulative incidence of infection with pandemic influenza H1N1 2009 was similar in various countries prior to vaccination. PLoS ONE. 6(8):21828.

3. Teresa del Rosal, Fernando Baquero-Artigao, Cristina Calvo, et al. (2011) Pandemic H1N1 influenza associated hospitalizations in children in Madrid, Spain. Influenza Other Respi Viruses. 5(6):544-551.

4. Halasa NB. (2010) Update on the 2009 pandemic influenza A H1N1 in children. Curr Opin Pediatr. 22(1):83-87.

5. Paules C, Subbarao K. (2017) Influenza. Lancet. 390(10095):697-708.

6. Centers for Disease Control and Prevention (CDC) Interim Guidance for Clinicians on the Prevention and Treatment of Swine-Origin Influenza Virus Infection in Young Children. (2009). 
7. Liolios L, Jenney A, Spelman D, et al. (2001) Comparison of a multiplex reverse transcription-PCR-enzyme hybridization assay with conventional viral culture and immunofluorescence techniques for the detection of seven viral respiratory pathogens. J Clin Microbiol. 39(8):2779-2783.

8. Mancinelli L, Onori M, Concato C, et al. (2016) Clinical features of children hospitalized with influenza A and B infections during the 2012-2013 influenza season in Italy. BMC Infect Dis. 16:6.

9. Quach C, Piché-Walker L, Platt R, et al. (2003) Risk Factors Associated With Severe Influenza Infections in Childhood: Implication for Vaccine Strategy. Pediatrics. 112(3 Pt 1);197.

10. Jané M, Vidal MJ, Soldevila N, et al. (2019) Epidemiological and clinical characteristics of children hospitalized due to influenza A and B in the south of Europe, 2010-2016. Scientific Reports. 9:12853.

11. Ostovar GA, Rubin LG, Rajan S, et al. (2011) Comparison of the Clinical Features of Children Hospitalized With Pandemic 2009 A:H1N1 and Seasonal Influenza. Clinical Pediatrics. 50(4):348-354. hospitalized with community acquired influenza. Pediatrics. 119(4):740-748.

13. Machablishvili A, Tsereteli D, Zakhashvili K, et al. (2017) Clinical and Epidemiological Characterization of Influenza A/H1n1pdm and B Among Hospitalized Children, Georgia, Season 2010-2011. Georgian Med News. 265:71-78.

14. Labeaud AD, Wentworth B, Gildengorin G, et al. (2013) Comparison Of Moderate And Severe Hospitalized Pediatric 2009 H1N1 Influenza Cases. Pediatr Infect Dis J. 32(2):90-93.

15. Shen CF, Huang SC, Wang SM, et al. (2008) Decreased leukocytes and other characteristics of laboratory findings of influenza virus infections in children. Microbiol Immunol Infect. 41(4):294-300.

16. M.P. Girard, J. Katz, Y. Pervikov, et al. (2010) Report of the 6th meeting on the evaluation of pandemic influenza vaccines in clinical trials World Health Organization, Geneva, Switzerland, 17-18 February. Vaccine. 28(42):6811-6820.

17. Huang C, Wang Y, Li X, et al. (2020) Clinical features of patients infected with 2019 novel coronavirus in Wuhan, China. Lancet. 395(10223):497-506.

12. Coffin SE, Zaoutis TE, Rosenquist AB, et al. (2007) Incidence, complications, and risk factors for prolonged stay in children 